\title{
LA DILIGENCIA POLICIAL DE VALORACIÓN DEL RIESGO DE VIOLENCIA DE GÉNERO EN EL SISTEMA VIOGÉN
}

\author{
Bárbara SÁNCHEZ LÓPEZ \\ Profesora Ayudante Doctora \\ Departamento de Derecho Procesal \\ y Derecho Penal Facultad de Derecho \\ Universidad Complutense de Madrid \\ basanche@ucm.es
}

\begin{abstract}
RESUMEN
El objeto de esta comunicación es la poco conocida diligencia de valoración del riesgo de violencia de género y su implementación en España a través del sistema $V d G$ o Viogén, cuyo desarrollo ba terminado configurando medidas policiales autónomas de protección de la víctima que pueden determinar el futuro de la valoración individual que el Estatuto de la Víctima de Delito, por exigencias de la Directiva 2012/29/UE, ba generalizado a toda clase de delitos violentos.
\end{abstract}

Palabras clave: proceso penal, funciones de la policía, estatuto de la víctima, violencia de género, valoración del riesgo de la víctima.

\section{ABSTRACT}

The purpose of this communique is the barely known diligence of an estimate of the risk of violence and its implementation in Spain through the VdG system or Viogen, whose development has ended up establishing local police measures for the protection of the victim which might settle the future of the individual estimate that the Statute for the Victim of Crime, following EU Directive 2012/29 has spread to all types of crimes with violence.

Keywords: penal procedure, police duties, statute of the victim, gender violence, estimate of the victim's risk.

\section{ZUSAMMENFASSUNG}

Das Ziel dieser Veröffentlichung ist das wenig bekannte Verfahren der Risikoeinstufung von geschlechtsspezifischer Gewalt und seine Implementierung in Spanien durch das System VdG oder Viogén vorzustellen. Die Entwicklung dieses Systems hat unabhängige polizeiliche Maßnabmen zum Schutz der Opfer hervorgebracht, welche die Zukunft der individuellen Risikenbewertung entscheidend bestimmen können. Sie debnen den Rechtsstatus des Straftatopfers, gemäß den Anforderungen der Richtlinie 2012/29/UE, allgemein auf alle Arten von Gewaltdelikten aus. Schlüsselwörter: strafverfahren, polizeiliche Funktionen, Rechtsstatus der opfer, geschlechtsspezifische Gewalt, Bewertung der Risiken des opfers. 
SUMARIO: I. EL PROTAGONISMO DE LAS FUERZAS DE SEGURIDAD DEL ESTADO EN LA DILIGENCIA DE VALORACIÓN INDIVIDUALIZADA DEL RIESGO DE VIOLENCIA DE GÉNERO.-II. EL SISTEMA VDG O VIOGÉN COMO PLATAFORMA DE SEGUIMIENTO INTEGRAL DE LA VIOLENCIA DE GÉNERO.-III. LA COMPILACIÓN DE LA INFORMACIÓN RELATIVA A LOS FACTORES DE RIESGO A TRAVÉS DE LOS FORMULARIOS VPR Y VPER.-IV. LA VALORACIÓN DEL RIESGO EN EL SISTEMA VIOGÉN Y LA ADOPCIÓN DE MEDIDAS DE PROTECCIÓN POLICIAL.-V. LA VALORACIÓN POLICIAL DE LA EVOLUCIÓN DEL RIESGO.

Desde el año 1999 en el que se introdujeron medidas de alejamiento en favor de las víctimas de violencia doméstica se han dado pasos de gigante en la implementación de medidas específicas de protección a las víctimas ${ }^{1}$. Por elementales razones de tiempo y de espacio no me detendré en desarrollar las medidas de alejamiento ni las órdenes de protección de víctimas, aunque — sin duda - han sido precursoras de la que aquí nos ocupa, que hasta hace pocos años ha presentado $-\mathrm{y}$ sigue presentando, pero no con carácter exclusivo- naturaleza instrumental respecto de las primeras. Me refiero a la llamada diligencia de valoración del riesgo de violencia de género y al conocido como «sistema VdG o Viogén», que apenas han concitado atención doctrinal desde el ámbito jurídico ${ }^{2}$ y, por ello, su conocimiento hoy suele estar restringido al círculo de profesionales de la psicología, la medicina forense o la policía que trabajan en este área ${ }^{3}$. Por su carác-

${ }^{1}$ Me refiero, fundamentalmente, a las Leyes Orgánicas 11/1999, de 30 de abril, y 14/1999, de 9 de junio, que introdujeron las medidas cautelares y definitivas de alejamiento en el proceso penal, y a la Ley 27/2003, de 31 de julio, reguladora de la orden de protección de las víctimas de la violencia doméstica, que fue el primer paso legislativo en materia de medidas específicas contra la violencia doméstica o en el ámbito familiar. La aspiración de una ley integral contra la violencia de género en especial se vio colmada apenas un año más tarde con la Ley Orgánica 1/2004, de 28 de diciembre, de Medidas de Protección Integral contra la Violencia de Género (LOMPIVG). El último hito del legislador nacional en materia de protección de la víctima está situado en la Ley 4/2015, de 27 de abril, reguladora del Estatuto de la Víctima del Delito, desarrollada mediante Real Decreto 1109/2015, de 11 de diciembre.

${ }^{2}$ Los estudios más reseñables, con perspectiva jurídica, se deben a M. J. García RodRíGUEZ, «Evaluación individual de las víctimas para determinar sus necesidades especiales de protección y asistencia en el marco del proceso penal», Revista General de Derecho Procesal, núm. 41 (2017), disponible en www.iustel.es, y a J. L. GonZÁlez ÁlvareZ, «Sistema de Seguimiento Integral de las Víctimas de Violencia de Género (Viogen)», en X Jornadas de la Asociación de Técnicos Superiores de Instituciones Penitenciarias. Almagro, 2016 (19, 20 y 21 de octubre), p. 102, disponible en https://www.icfs.es/wp-content/uploads/2017/07/libroatip-2016-web.pdf.

${ }^{3}$ Desde estas otras importantes perspectivas destacan las aportaciones de M. J. GARRIDo ANTÓN, Validación del procedimiento de valoración del riesgo de los casos de violencia de 
ter multidisciplinar y, sobre todo, por la importancia que va a cobrar en el futuro con la generalización de la diligencia de valoración del riesgo a toda clase de delitos violentos ${ }^{4}$, merece la pena que nos detengamos unos minutos en conocer en qué consiste esta valoración individual del riesgo de violencia de género por parte de la policía.

\section{EL PROTAGONISMO DE LAS FUERZAS DE SEGURIDAD DEL ESTADO EN LA DILIGENCIA DE VALORACIÓN INDIVIDUALIZADA DEL RIESGO DE VIOLENCIA DE GÉNERO}

La LOMPVIG proporciona el marco legal difuso - pero, al parecer, suficiente y, en todo caso, sobrevenidamente consolidado por la LECDde esta medida consistente en la valoración del riesgo de la víctima de violencia de género. Ningún artículo se ocupa de ella frontalmente, sino que es el resultado de la coordinación entre las diferentes Administraciones Públicas competentes en materia de prevención, detección e intervención continuada en favor de la mujer en situación de riesgo de padecer violencia de género, con base en los diferentes artículos que regulan sus funciones (vid., en lo referente a la policía, el art. 32 LOMPVIG). Y es que, aunque cabría imaginar que la valoración de esta situación de riesgo sería una tarea exclusiva de los jueces con la participación de las Unidades de Valo-

\footnotetext{
género del Ministerio del Interior de España, tesis doctoral inédita, Departamento de Psicología Biológica y de la Salud de la Universidad Autónoma de Madrid, 2012, disponible en http://hdl.handle.net/10486/11170; J. ZuRITA BAYONA, Violencia contra la mujer. Marco bistórico evolutivo y predicción del nivel de riesgo, tesis doctoral inédita, Departamento de Psicología Biológica y de la Salud de la Universidad Autónoma de Madrid, 2014, disponible en http://hdl.handle.net/10486/661810, y J. J. LóPEZ-Ossorio, Construcción y validación de los formularios de valoración policial del riesgo de reincidencia y violencia grave contra la pareja (VPR4.0-VPER4.0) del Ministerio del Interior de España, tesis doctoral inédita, Departamento de Psicología Biológica y de la Salud de la Universidad Autónoma de Madrid, 2017. Cfr. también J. J. López-Ossorio, J. L. González-Álvarez y A. Andrés-Pueyo, «Eficacia predictiva de la valoración policial del riesgo de la violencia de género», Psychosocial Intervention, vol. 25, núm. 1 (2016), pp. 1-7, disponible en bttp://www.redalyc.org/ pdf/1798/179844973001.pdf.

${ }_{4}$ Vid. art. 22 de la Directiva 2012/29/UE del Parlamento Europeo y del Consejo, de 25 de octubre de 2012, por la que se establecen normas mínimas sobre los derechos, el apoyo y la protección de las víctimas de delitos, y por la que se sustituye la Decisión Marco 2001/220/JAI del Consejo; los arts. 23-24 de la Ley 4/2015, de 27 de abril, reguladora del Estatuto de la Víctima del Delito (en adelante, LEVD), y su desarrollo en los arts. 9, 30 y ss. del Real Decreto 1109/2015, de 11 de diciembre, por el que se desarrolla la LEVD y se regulan las Oficinas de Asistencia a las Víctimas del Delito.
} 
ración Forense Integral a que se refiere la disposición adicional segunda LOMPIVG, en ningún lugar está dicho que se trate de una medida exclusivamente jurisdiccional. Más aun, si el objetivo en esta materia es proporcionar una respuesta inmediata a las necesidades de seguridad de la víctima, no conviene — probablemente- que la valoración del riesgo y la adopción inmediata de medidas adecuadas hayan de ser siempre jurisdiccionales, habida cuenta que la primera respuesta institucional la proporcionan las Fuerzas de Seguridad del Estado (en adelante, FSE). Lo que no cabe en ningún caso es excluir el control jurisdiccional sobre las diligencias de la policía o de cualquier otra Administración, sean de la clase que sean.

En la actualidad, la valoración policial individualizada del riesgo para toda víctima está expresamente contemplada — desde el año 2015- en el art. 282 LECrim., reformado por la Ley del Estatuto de la Víctima del Delito (LEVD) ${ }^{5}$. Pero esta disposición no existía al tiempo de promulgarse la LOMPIVG. Su implantación en materia de violencia de género mediante la plataforma que hoy conocemos como sistema VdG o Viogén ha venido propiciada por varios hechos: 1) por la creación (ex art. 31 LOMPIVG) de unidades policiales «especializadas en la prevención de la violencia de género y en el control de la ejecución de las medidas judiciales adoptadas en materia de violencia de género» ${ }^{6}$, y 2 ) por la aprobación de sucesivos

${ }^{5}$ Dice ahora el art. 282 LECrim.: «La policía judicial tiene por objeto y será obligación de todos los que la componen, averiguar los delitos públicos que se cometieren en su territorio o demarcación; practicar, según sus atribuciones, las diligencias necesarias para comprobarlos y descubrir a los delincuentes, y recoger todos los efectos, instrumentos o pruebas del delito de cuya desaparición hubiere peligro, poniéndolos a disposición de la autoridad judicial. Cuando las víctimas entren en contacto con la policía judicial, cumplirá con los deberes de información que prevé la legislación vigente. Asimismo, llevarán a cabo una valoración de las circunstancias particulares de las víctimas para determinar provisionalmente qué medidas de protección deben ser adoptadas para garantizarles una protección adecuada, sin perjuicio de la decisión final que corresponderá adoptar al juez o tribunal.

Si el delito fuera de los que solo pueden perseguirse a instancia de parte legítima, tendrán la misma obligación expresada en el párrafo anterior, si se les requiere al efecto. La ausencia de denuncia no impedirá la práctica de las primeras diligencias de prevención y aseguramiento de los delitos relativos a la propiedad intelectual e industrial».

6 Así, en el Cuerpo Nacional de Policía la valoración se realiza por las Unidades de Familia y Mujer (UFAM), que prestan una atención integral, especializada y personalizada a las víctimas de violencia de género, violencia doméstica y violencia sexual [integran a las antiguas Unidades de Prevención, Asistencia y Protección a la mujer maltratada (UPAP) y los Servicios de Atención a la Mujer (SAM)]. Por su parte, en la Guardia Civil existen unidades específicas llamadas Equipo Mujer-Menor (EMUME), creadas en 1995 como equipos especializados en hechos delictivos en los que se encuentran implicados mujeres y menores, tanto en calidad de víctimas como de autores; trabajan en Puntos de Atención Especializada (PAE) comarcales y provinciales, y a nivel nacional trabaja el EMUME-Central, que presta labores de apoyo y que interviene directamente cuando concurren circunstancias de especial 
protocolos de actuación y coordinación policiales en materia de violencia de género que han tenido un efecto de refuerzo sobre la función de la policía en la prevención y detección de las situaciones de riesgo para la víctima ${ }^{7}$. Como corolario de estas medidas, dentro del catálogo de medidas urgentes del Plan Nacional de Sensibilización y Prevención de la Violencia de Género 2007-2008, se incluyó la implantación del «sistema de seguimiento integral en los casos de violencia de género, sistema VdG o Viogén» ${ }^{8}$.

De esta forma, desde el mismo momento en que la policía tenga conocimiento de hechos que pudieran ser constitutivos de infracción penal en materia de violencia de género, las FSE han de practicar - como diligencia de investigación que debe incorporarse al atestado- ${ }^{9}$ las medidas de averiguación de la existencia e intensidad de la situación de riesgo para la víctima, fundamentalmente a través de la toma de declaración de la víctima, de testigos, pero también recabando información de personas del entorno familiar, vecinal, laboral, servicios sociales, servicios sanitarios y administrativos de atención a la víctima, consultas en los registros públicos, etc. La finalidad de la información recopilada radica en acordar las medidas más adecuadas al nivel de riesgo detectado y, por eso, su eficacia reside en la puesta en común de esta información a través de esta plataforma VdG o Viogén, que está en funcionamiento desde julio de 2007.

complejidad. Y, por último, en los ámbitos autonómico y municipal, un número creciente de administraciones han creado también unidades especializadas que colaboran con los Juzgados y con los servicios sociales. A nivel autonómico merece destacar, por su integración en el sistema Viogén, la especialización de la Policía Foral de Navarra. En el escalón municipal, por ejemplo, en la Villa de Madrid asume la función de policía especializada la Unidad de Atención y Protección a la Familia (UAPF).

${ }^{7} \mathrm{La}$ decena de protocolos policiales aplicables a esta materia pueden consultarse en http://www.poderjudicial.es/cgpj/es/Temas/Violencia-domestica-y-de-genero/Guias-yProtocolos-de-actuacion/Protocolos/. Nosotros centraremos nuestra atención en el nuevo protocolo del año 2016 para la valoración policial del nivel de riesgo de violencia de género.

${ }^{8}$ El Consejo de Ministros de 22 de junio de 2007 aprobó estas medidas, dentro de las cuales consta la creación de una «Aplicación informática que permite reunir todos los datos de que disponen las Fuerzas y Cuerpos de Seguridad del Estado e Instituciones Penitenciarias. Instrumento para la valoración del riesgo. Aplicación del sistema de control GPS a los agresores», disponible en http://www.lamoncloa.gob.es/consejodeministros/ referencias/Paginas/2007/refc20070622.aspx\#ViolenciaG por 100C3 por 100A9nero. Mediante Orden INT/1911/2007, de 26 de junio, ya derogada, se creó en el Ministerio del Interior el fichero de datos de carácter personal «Violencia doméstica y de género».

9 Como hace notar M. DE Hoyos SANChO, «Particularidades de la investigación y prueba de los delitos de violencia doméstica y de género», en C. Guilarte Martín-CaLERO (coord.), Aspectos civiles y penales de las crisis matrimoniales, Valladolid, Lex Nova, 2009, p. 164, nota 12, en sede judicial debe ser posible contrastar la información con informes periciales de las unidades de valoración forense integral. 


\section{EL SISTEMA VDG O VIOGÉN COMO PLATAFORMA DE SEGUIMIENTO INTEGRAL DE LA VIOLENCIA DE GÉNERO}

El sistema VdG o Viogén, de seguimiento integral en los casos de violencia de género, es un servicio estratégico que se mantiene constantemente actualizado y que integra, tecnológicamente, toda la información disponible sobre un caso o sobre una víctima en los que se ha realizado ya la valoración del riesgo ${ }^{10}$. Los casos salen del sistema si causan baja por haberse dictado con carácter firme sentencia absolutoria o auto de sobreseimiento libre del encausado, o por cumplimiento y cancelación de antecedentes (o por fallecimiento). En la fecha actual de principios de mayo de 2018 existen en el sistema VdD o Viogén casi medio millón de casos, que comprenden tanto los casos activos (los que son objeto de atención policial en términos actuales) como los inactivos (casos que han sido activos y que no lo son en términos actuales, pero que son susceptibles de volver a serlo). En la actualidad, el número de casos activos representa el 10,90 por 100 de todos los casos que gestiona el sistema ${ }^{11}$.

La información procede de las declaraciones de la propia víctima, de los testigos directos y de referencia, y de las incidencias provenientes del Registro Judicial de Medidas Cautelares, de dispositivos de geoposicionamiento, de servicios de atención y asistencia para víctimas, del Registro de Armas, de antecedentes policiales y - tratándose de agentes de la Policía Nacional y la Guardia Civil— de cuantos ficheros policiales pueden consultar (como, por ejemplo, la situación legal en España de personas extranjeras mediante ADEXTRA). Las finalidades que cumple el sistema Viogén son, fundamentalmente, tres: 1) integrar toda la información policial, judicial y penitenciaria sobre la violencia de género para coordinar las actuaciones necesarias; 2) realizar la valoración del nivel de riesgo de padecer nuevas agresiones y así controlar su seguimiento, y 3) proporcionar esta información a los agentes institucionales que intervienen en el área de la

${ }^{10}$ En el sistema Viogén un caso agrupa toda información que vincula a una víctima con un agresor determinado; si la víctima ha padecido violencia por parte de más de un agresor, cada uno de ellos genera un caso. El sistema Viogén no integra los casos que tienen pendiente la realización de la valoración del riesgo.

${ }^{11}$ El número total de casos que gestiona Viogén es de 497.641 según el Informe estadístico del sistema Viogén con datos tomados al 30 de abril de 2018. De ellos, son casos activos 54.259 y solo 500 han causado baja. El resto son casos inactivos. 
violencia de género. Integra, además, un subsistema muy útil llamado «sistema 3A: Aviso, Alerta, Alarma», que genera alertas y notificaciones telemáticas inmediatas sobre ciertas circunstancias significativas (por ejemplo, para informar a la víctima sobre los cambios en la situación de libertad o de prisión del agresor, para avisar a los agentes de la necesidad de revisar la evolución del riesgo, para alertarles de que se ha reanudado la convivencia de la víctima con el agresor, de que se ha registrado una nueva agresión de un autor con otra víctima o de la suspensión o finalización de una orden de protección). Con la implementación de este subsistema se consigue una permanente actualización del nivel de riesgo de la víctima que se denomina «Estimación Permanente de Evolución del Riesgo» (EPER).

El servicio entró en funcionamiento a raíz de una primera Instrucción núm. 10/2007, de 10 de julio, de la Secretaría de Estado de Seguridad del Ministerio del Interior, por la que se aprobó el «Protocolo para la valoración policial del nivel de riesgo de violencia contra la mujer en los supuestos de la Ley Orgánica 1/2004, de 28 de diciembre, y su comunicación a los órganos judiciales y al Ministerio Fiscal». En la actualidad y tras diversas modificaciones, tanto del protocolo como de los formularios de valoración de riesgo, el protocolo ha sido sustituido por otro aprobado mediante Instrucción 7/2016, de la Secretaría de Estado de Seguridad (en adelante, «el Protocolo»), que asegura la uniformidad en la compilación y el tratamiento de los datos y su fácil consulta por las personas autorizadas. El acceso al fichero de datos y su gestión se rige por la vigente Orden INT/1202/2011, de 4 de mayo (vid. anexo II.13: fichero de violencia doméstica y de género).

El alto nivel de seguridad en el acceso al fichero explica que solo puedan acceder a él determinados usuarios institucionales y que, mediante su identificación electrónica, los usuarios gocen de diferentes niveles de acceso y edición de los datos. Pueden acceder a él los órganos judiciales del orden penal, los fiscales destinados en las fiscalías de los tribunales competentes, los funcionarios autorizados de las unidades policiales especializadas en violencia de género, los directores de los centros penitenciarios o de los centros de inserción social, delegaciones y subdelegaciones del Gobierno, las Unidades de Valoración Forense Integral y el personal de las Comunidades Autónomas, y previa suscripción del correspondiente convenio de cooperación con la Secretaría de Estado de Seguridad del Ministerio del Interior, el personal de las entidades locales con competencia en servicios asistenciales, puntos de coordinación de las órdenes de protección de violencia doméstica y de género, y oficinas de atención a la víctima del delito. 


\section{LA COMPILACIÓN DE LA INFORMACIÓN RELATIVA A LOS FACTORES DE RIESGO A TRAVÉS DE LOS FORMULARIOS VPR Y VPER}

Como diligencia policial, los agentes especializados deben compilar la información imprescindible para evaluar tanto el riesgo de que se produzca una nueva agresión [Valoración Policial del Riesgo (VPR)] como su evolución posterior [Valoración Policial de la Evolución del Riesgo (VPER)]. La recogida de esta información se realiza por los agentes policiales - nunca por la víctima- cumplimentando los factores de riesgo de tipo histórico que permiten realizar la estimación del riesgo a través de dos formularios distintos y que van ya — desde septiembre de 2016 - por la novedosa versión $4.0^{12}$.

La información inicial del caso se compila a través del «formulario $\mathrm{VPR}$ », en el que se contemplan treinta y nueve indicadores de riesgo. Algunos de ellos son, por ejemplo, si han existido vejaciones, insultos, humillaciones, violencia física o sexual; cuáles han sido las reacciones defensivas de la víctima; si el agresor ha empleado armas contra la víctima y si tiene acceso a ellas; si la víctima ha recibido amenazas o planes para causarle daño físico o psíquico; si ha existido una escalada en la gravedad o en la frecuencia de las agresiones o amenazas; si el agresor ha mostrado en los últimos seis meses celos exagerados o conductas de control sobre la víctima; si el agresor ha dado muestras de comportamiento violento causando daños materiales, o faltando el respeto a la autoridad, o agrediendo a personas o animales, o despreciando o enfrentándose a terceras personas; si en los últimos seis meses existen indicios de que el agresor tenga algún problema laboral o de otro orden distintos de la relación de pareja; si el agresor se encuentra fugado o en paradero desconocido.

En la versión inmediatamente anterior a la actual había que indicar — respecto de cada ítem de riesgo- el o las varias fuentes de la infor-

${ }_{12}$ El desarrollo inicial de estos formularios se debe al trabajo conjunto de especialistas policiales y de investigadores del área de la psicología procedentes de la Universidad Autónoma de Madrid, de la Universidad de Barcelona y de la Universidad de Málaga. En la actual versión 4.0, efectuada tras una revisión de la eficacia predictiva del sistema, han trabajado durante más de dos años un grupo de trabajo de expertos del Ministerio de Interior, la magistratura, el Consejo General del Poder Judicial, la Fiscalía Especial de Violencia sobre la Mujer, la Delegación del Gobierno para la Violencia de Género y de las FSE. Cfr. M. J. GARCíA RodríGUEZ, «Evaluación individual de las víctimas...», op. cit., p. 747, y J. L. GonZÁLEZ ÁlvareZ, «Sistema de Seguimiento Integral de las Víctimas...», op. cit., p. 111. 
mación mediante opciones predeterminadas acumulables («víctima», «autor», «testigo», «informe técnico») y, al mismo tiempo, se debía seleccionar el nivel de riesgo apreciado entre las seis opciones disponibles: «no sabe», «no disponible», «bajo», «medio», alto»y «extremo» (este último introducido en el año 2008). La versión actual sigue compilando las varias fuentes, pero con la mayor concreción de los indicadores de riesgo, la respuesta al nivel de riesgo apreciado se ha ajustado a un formato más concreto y polarizado: «sí̀, «no» o «no (se) sabe».

\section{LA VALORACIÓN DEL RIESGO EN EL SISTEMA VIOGÉN Y LA ADOPCIÓN DE MEDIDAS DE PROTECCIÓN POLICIAL}

Los datos así recogidos se someten a algoritmos diseñados ad hoc para valorar automáticamente las puntuaciones introducidas en los distintos ítems. Estos algoritmos son producto del estudio continuado de los policías, psicólogos y académicos expertos que trabajan en el desarrollo del sistema Viogén en la Secretaría de Estado de Seguridad.

El sistema devuelve la valoración de riesgo de la víctima estructurada en los siguientes cinco niveles de riesgo: «no apreciado» (25.933 casos), «bajo» (23.357 casos), «medio» (4.767 casos), «alto» (188 casos) y «extremo» (14 casos). Este nivel puede ser variado al alza por los agentes actuantes en atención a circunstancias que no vengan recogidas en los indicadores del sistema y siempre que lo valoren como necesarias para la mejor protección de la víctima.

Cada uno de los niveles de riesgo lleva aparejadas determinadas medidas policiales de protección y seguridad de las víctimas de carácter obligatorio y de carácter complementario (que se encuentran recogidas en el Adjunto I del nuevo Protocolo), así como —novedosamente con el nuevo Protocolo- medidas de autoprotección por parte de la víctima (Adjunto II del Protocolo), que constituyen el «Plan de seguridad personalizado de cada víctima», con especial atención a víctimas menores (Adjunto III del Protocolo). Por lógicas razones de brevedad no puedo extenderme en explicar las medidas asociadas a cada nivel de riesgo, aunque no puedo dejar de referirme, cuanto menos, a las medidas previstas para los dos niveles de riesgo más frecuentes: el nivel de riesgo «no apreciado», que es el nivel prevalente con 25.933 casos en la actualidad, y el nivel de riesgo «bajo», que sigue en número con 23.357 casos. También 
he de hacer notar que las medidas asociadas a un nivel de riesgo supone la adopción de las previstas para todos los niveles de riesgo menores que el valorado.

Cuando la valoración del riesgo de violencia de género que arroja Viogén es «no apreciado», corresponde adoptar las medidas de información y prevención aplicables a cualquier ciudadano para informarle de los derechos y de los recursos públicos a su disposición y ofrecerle recomendaciones de autoprotección, complementadas con la facilitación de los teléfonos de emergencia y la asistencia especializada.

Cuando, en un escalón superior, el nivel de riesgo es «bajo», la policía debe adoptar, de un lado, medidas obligatorias consistentes en: 1) respecto del agresor, comunicarle que la víctima dispone de un servicio policial de protección y requerirle - si tuviera licencia de armas- para que las entregue voluntariamente a los agentes actuantes, cursando posteriormente una solicitud de retirada del permiso de armas a la autoridad judicial, y 2) respecto de la víctima, establecer contactos telefónicos esporádicos con ella; facilitarle números de teléfono de contacto permanente (veinticuatro horas) con las FSE; informarle de forma precisa sobre el servicio de teleasistencia móvil; derivarle hacia los servicios sociales y asistenciales que correspondan a su domicilio, recomendándole encarecidamente que se informe de los recursos a su disposición (especialmente los que tengan que ver con su seguridad: puntos de encuentro, viviendas de acogida, etc.), y darle recomendaciones sobre autoprotección y modos de evitar incidentes. Además, y como medidas complementarias de protección correspondientes a este nivel, los agentes pueden establecer contactos personales, esporádicos y discretos, con la víctima; confeccionar una ficha con los datos relevantes de la víctima y del agresor para que la porte el personal de patrulla, y acompañar a la persona denunciada — si el juez ha acordado su salida del domicilio- a recoger sus enseres. Lo anterior se completa con el diseño del Plan de Seguridad de la Víctima, que en este nivel incluye las recomendaciones de: 1) portar siempre el teléfono móvil con la lista de números de teléfono importantes y de emergencia en un lugar preferente, así como guardar los números de emergencia (112, 091, 062 y 092) en el dispositivo móvil vinculándolos a teclas de marcación automática y rápida; 2 ) instalar en el móvil la app AlertCops, una aplicación telemática de seguridad ciudadana para dispositivos móviles desarrollada por el Ministerio de Interior, que presta un servicio de notificación inmediata de denuncias, u otra aplicación equivalente, y 3 ) fomentar la realización de cursos de defensa personal. 
El resultado de la VPR se comunica al Juzgado y, en su caso, al Ministerio Fiscal junto en un informe que se incluye en el atestado y debe recoger los principales factores de riesgo apreciados. Judicializado el atestado, corresponde al juez valorar, con el auxilio de las Unidades de Valoración Forense Integral, las circunstancias fácticas que justifican la adopción de medidas cautelares de protección de la víctima, a cuyo efecto el informe policial constituye una diligencia más que permite su más precisa valoración judicial.

Cabe, así, que existan discrepancias entre las medidas policiales implantadas y la resolución judicial sobre las medidas cautelares procedentes. Para el caso de discrepancia, el Protocolo, no obstante reconocer la preeminencia de las medidas judiciales que en su caso se acuerden, impone el deber de las FSE de «seguir facilitando la protección que corresponda a la víctima por su nivel de riesgo», y el caso permanecerá «activo» en el Sistema Viogén hasta que el nivel de riesgo llegue a «no apreciado» y se pase a «inactivo». Y si es imposible el seguimiento de estas medidas policiales que cabe calificar como autónomas a causa, por ejemplo, de renunciar la víctima a una orden de protección o por trasladarse al extranjero, «estas se adaptarán a las circunstancias que se deriven de la nueva situación».

\section{LA VALORACIÓN POLICIAL DE LA EVOLUCIÓN DEL RIESGO}

Por lo demás, como el riesgo de padecer un episodio de violencia de género es siempre cambiante y dinámico, una vez efectuada la valoración inicial del riesgo corresponde mantener actualizada la evaluación del mismo. De ahí que el Protocolo imponga la obligación de monitorizar la situación de riesgo de la víctima y realizar la VPER en tres supuestos: cuando lo pida el juez o el Ministerio Fiscal, cuando se comunique algún hecho o cambio significativo (por ejemplo, una nueva denuncia o el quebrantamiento de una medida de protección) o, periódicamente, mediante una reevaluación de los factores de riesgo y como parte de la función de predicción y prevención de la violencia de género que cumple el sistema Viogén.

La VPER se completa también telemáticamente a través de ítems de riesgo que permiten valorar circunstancias sobrevenidas desde la última evaluación, para lo que pueden resultar precisas nuevas entrevistas con la víctima y con personas de su entorno. Una vez que un caso es registrado en 
Bárbara Sánchez López La diligencia policial de valoración del riesgo de violencia...

el sistema Viogén es sometido a la realización periódica de la VPER cada cierto tiempo predeterminado que es inversamente proporcional al nivel de riesgo apreciado en la valoración de la que se parte. Así, el nivel extremo ha de ser reevaluado antes de setenta y dos horas; el nivel alto, antes de siete días; el nivel medio, antes de treinta días; el bajo, antes de sesenta días, y el nivel no apreciado, cada sesenta días cuando la víctima cuente con orden de protección y cada noventa días si no cuenta con orden de protección.

Cuando se estime, en fin, que han desaparecido o remitido las circunstancias de riesgo para la víctima, el Protocolo establece que las FSE lo comuniquen al Juzgado, «informando sobre los factores determinantes de tal valoración, pasando el caso a estar "inactivo" en el sistema Viogén, cuando no resulte de aplicación una medida cautelar de protección en vigor». 